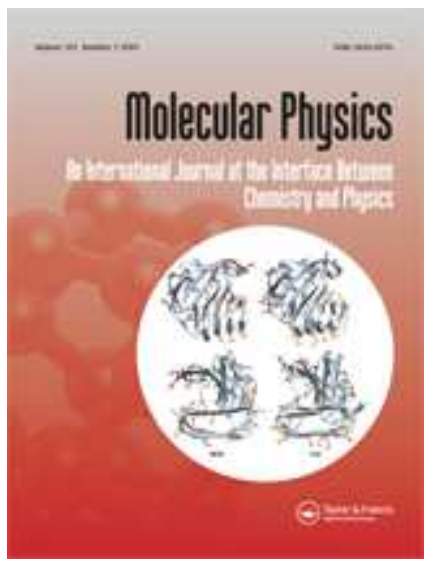

\title{
Local polarizabilities and dispersion energy coefficients
}

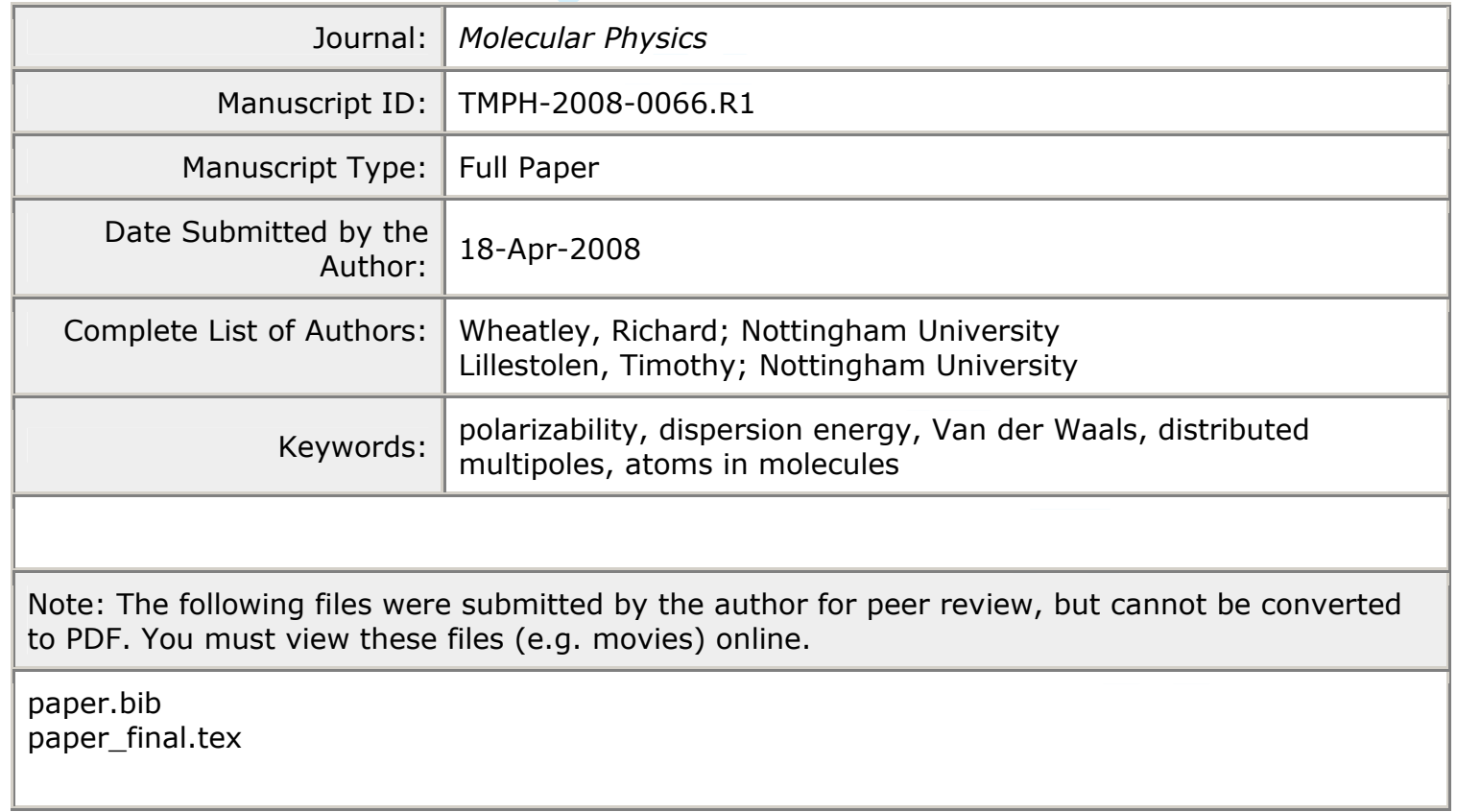

\section{S) ScholaroNE \\ Manuscript Central}




\title{
Local polarizabilities and dispersion energy coefficients.
}

\author{
Richard J. Wheatley* and Timothy C. Lillestolen \\ School of Chemistry, \\ The University of Nottingham, Nottingham, \\ United Kingdom, NG7 2RD
}

\begin{abstract}
Static and dynamic polarizabilities of atoms and functional groups within molecules are calculated from the response of the distributed multipoles to different applied potentials, using a newly developed localisation scheme. The methodology, described here in full for the first time, is based entirely on first-principles calculations. It gives physically reasonable and transferable polarizabilities and dispersion energy coefficients, for multipole components up to at least octopole. Polarizabilities and dispersion energy coefficients determined using this method are used to calculate induction and dispersion energies which are compared with $a b$ initio energies calculated at the Coupled Hartree-Fock level of theory. The localized polarizabilities and dispersion energy coefficients are shown to be an improvement over the corresponding molecular values, in terms of both absolute accuracy as well as convergence properties.
\end{abstract}

Key words: polarizability, dispersion energy coefficient, Van der Waals, atoms in molecules, distributed multipole analysis

PACS numbers:

*Electronic address: Richard.Wheatley@nottingham.ac.uk 


\section{INTRODUCTION}

Computer simulations of molecules in the liquid and solid state and in clusters rely for their predictive power on a reasonable representation of the intermolecular interactions. With the continuing rapid increase in computational power, molecular simulations can now use more elaborate and realistic intermolecular potential energy functions, and theoretical methods for calculating these intermolecular potentials are advancing rapidly [1]. The starting point for developing models of intermolecular interactions is usually the long-range multipole coefficients $C_{n}$, which describe interactions between well separated molecules, and, for all but the smallest molecules, these multipole coefficients must be known for local regions of a molecule, such as the individual atoms or united atoms, not merely for the molecule as a whole. Local $C_{n}$ coefficients can be calculated from local multipoles and from static and dynamic local polarizabilities. Physically reasonable and useful local multipoles are relatively straightforward to obtain from first principles, and a number of methods have been published [2-6], but local polarizabilities are much more difficult to obtain, mainly because of the inherent non-locality involved in the response of the charge density of a molecule to an applied electrostatic potential. Nevertheless, the potential rewards for obtaining local polarizabilities (in particular, a first-principles method for obtaining atom-atom dispersion energy coefficients) have stimulated much work in this area [7-15], with many of these developments being made by the Stone group.

We recently [16] introduced a new method for calculating local polarizabilities, and gave results for $l=1$ to 3 (dipole to octopole polarizabilities) for several small molecules and hydrocarbons up to hexane. The method has a combination of features which distinguishes it from previous work in this area. It requires only first-principles electronic structure calculations, and no fitting is involved. The calculated polarizabilities are local to the separate atoms or functional groups, their values appear to be reasonable, and the net effect of all the separate local polarizabilities reproduces the total molecular polarizabilities. The polarisation energies of molecules in different electrostatic potentials converge more quickly with increasing multipole rank when they are calculated using these local polarizabilities, than when they are calculated using the total molecular polarizabilities.

However, our published method is incomplete for two main reasons. It relies on information about 'bonds' between atoms, which would not be available in a truly first-principles 


\section{THEORY}

Polarizabilities $\alpha$ describe the response of the charge density $\rho$ of a molecule $A$ to an applied electrostatic potential $V$. If the applied potential obeys Laplace's equation then it can be expanded in a multipole series about an origin of coordinates, whose location is immaterial in what follows:

$$
V=\sum_{\lambda} V_{\lambda} R_{\lambda}(\mathbf{r})
$$

where $\lambda$ denotes angular momentum $(l, m), R_{\lambda}=C_{\lambda} r^{l}$ is a regular spherical harmonic function, and $C$ is an unnormalised spherical harmonic function. (Applied potentials that do not obey Laplace's equation require a more elaborate treatment than is provided by polarizabilities, so they are not considered further here.) The applied potential changes the 
molecular multipoles $Q$, which are given by

$$
Q_{\lambda}=\int \rho(\mathbf{r}) R_{\lambda}(\mathbf{r}) d \mathbf{r}
$$

where $\rho$ is the charge density. The molecular polarizabilities are defined as the linear response of the multipole moments to the applied potential:

$$
\alpha_{\lambda, \lambda^{\prime}}=-\left(\frac{\partial Q_{\lambda}}{\partial V_{\lambda^{\prime}}}\right)_{V=0},
$$

and $\alpha_{\lambda, \lambda^{\prime}}=\alpha_{\lambda^{\prime}, \lambda}$ (the polarizability is symmetrical) because $Q_{\lambda}=\left(\partial E / \partial V_{\lambda}\right)_{V=0}$, where $E$ is the electronic energy.

Local multipoles $Q_{a, \lambda}$ for the atoms or functional groups $a$ within a molecule cannot be defined uniquely. In this work we use the Distributed Multipole Analysis (DMA) method [2], and the recently modified DMA method [6] which reduces the basis set dependence of the DMA multipoles. However, our method for calculating local polarizabilities can be applied to any set of local multipoles, provided that they reproduce the total molecular multipoles up to the same maximum multipole rank:

$$
Q_{\lambda}=\sum_{a} \sum_{\lambda^{\prime}} Q_{a, \lambda^{\prime}} T_{\lambda, \lambda^{\prime}}\left(\mathbf{r}_{a}\right)
$$

where $\mathbf{r}_{a}$ is the location of the nucleus of atom $a$, or the centre of functional group $a$, and the transfer functions $T$ are defined by the expansion of a regular spherical harmonic function about a different origin [2]:

$$
R_{\lambda}\left(\mathbf{r}-\mathbf{r}_{a}\right)=\sum_{\lambda^{\prime}} T_{\lambda, \lambda^{\prime}}\left(\mathbf{r}_{b}-\mathbf{r}_{a}\right) R_{\lambda^{\prime}}\left(\mathbf{r}-\mathbf{r}_{b}\right)
$$

This series terminates, and $T_{\lambda, \lambda^{\prime}}=0$ if $l<l^{\prime}$. In other words, the first subscript of any non-zero function $T$ has multipole rank greater than or equal to the second subscript.

Now we consider the effect on the local multipoles of applying a potential to the molecule. The linear response is denoted by $\Pi^{u}$, where

$$
\Pi_{a, \lambda, \lambda^{\prime}}^{u}=-\left(\frac{\partial Q_{a, \lambda}}{\partial V_{\lambda^{\prime}}}\right)_{V=0}
$$

and the superscript $u$ denotes 'uncorrected', for reasons which are explained below. The response $\Pi_{a, \lambda, \lambda^{\prime}}^{u}$ is not usually symmetric with respect to exchange of its angular momentum subscripts $\lambda$ and $\lambda^{\prime}$, and, since $V_{\lambda^{\prime}}$ is a molecular (not local) potential, $\Pi_{a, \lambda, \lambda^{\prime}}^{u}$ is not usually 
a local polarizability. Local polarizabilities $\alpha_{a}$ may be defined by analogy with molecular polarizabilities:

$$
\alpha_{a, \lambda, \lambda^{\prime}}=-\left(\frac{\partial Q_{a, \lambda}}{\partial V_{a, \lambda^{\prime}}}\right)_{V=0}
$$

where $V_{a, \lambda^{\prime}}$ is a multipole component of the applied potential with respect to $\mathbf{r}_{a}$, the centre of local region $a$. From equations (1) and (5) it follows that

$$
V_{a, \lambda^{\prime}}=\sum_{\lambda} V_{\lambda} T_{\lambda, \lambda^{\prime}}\left(\mathbf{r}_{a}\right)
$$

and combining this with equations (6) and (7) gives

$$
\Pi_{a, \lambda, \lambda^{\prime}}^{u}=\sum_{\lambda^{\prime \prime}} \alpha_{a, \lambda, \lambda^{\prime \prime}}^{u} T_{\lambda^{\prime}, \lambda^{\prime \prime}}\left(\mathbf{r}_{a}\right)
$$

A superscript $u$ (uncorrected) is attached to the local polarizability $\alpha_{a}^{u}$ in this equation, as this definition of local polarizability will be modified below. From the properties of the $T$ functions it follows that the sum can be restricted to $l^{\prime \prime} \leq l^{\prime}$, where $l^{\prime}$ is the multipole rank of the applied potential.

Since the linear responses $\Pi^{u}$ can be calculated from first principles (given a suitable method for obtaining local multipoles), the local polarizabilities can be obtained from equation (9), if the reasonable assumption is made that they are symmetrical: $\alpha_{a, \lambda, \lambda^{\prime}}^{u}=\alpha_{a, \lambda^{\prime}, \lambda}^{u}$. The calculation is done for increasing values of $l^{\prime}$ and $l$, first considering $l^{\prime}=0$ and all $l \geq 0$ in equation (9), then $l^{\prime}=1$ and all $l \geq 1$, and continuing up to any required maximum multipole rank. For example, consider the $m=0$ components of the polarizability of a linear molecule aligned along the $\mathrm{z}$ axis. Using the explicit expressions for $T$ in equation (9) produces, for $l^{\prime}=0$,

$$
\Pi_{a, l 0,00}^{u}=\alpha_{a, l 0,00}^{u}
$$

and the left hand side is zero, because applying a constant potential $V_{00} R_{00}$ to a molecule has no effect on its charge density, so the polarizabilities on the right hand side are all zero, which is an essential requirement for local polarizabilities. The assumption of symmetry then gives $\alpha_{a, 00, l 0}^{u}=0$ for all $l$.

Applying a constant electric field gives $l^{\prime}=1$ in equation (9), and there are two terms on the right hand side:

$$
\Pi_{a, l 0,10}^{u}=\alpha_{a, l 0,00}^{u} z_{a}+\alpha_{a, l 0,10}^{u}
$$


where $z_{a}$ is the z-coordinate of atom/region $a$. Since the 'charge polarizabilities' are zero, only the second term on the right hand side remains, so all the polarizabilities $\alpha_{a, l 0,10}^{u}$, and hence $\alpha_{a, 10, l 0}^{u}$, can be obtained.

Next, a constant electric field gradient $l^{\prime}=2$ is applied. Eliminating zero polarizabilities from the right hand side of equation (9) gives

$$
\Pi_{a, l 0,20}^{u}=\alpha_{a, l 0,10}^{u}\left(2 z_{a}\right)+\alpha_{a, l 0,20}^{u} .
$$

Since the polarizabilities $\alpha_{a, l 0,10}^{u}$ were obtained in the previous step, this equation yields the quadrupole polarizabilities $\alpha_{a, l 0,20}^{u}=\alpha_{a, 20, l 0}^{u}$.

However, this simple procedure does not give symmetrical local polarizabilities. For example, equation (11) with $l=0$ gives $\Pi_{a, 00,10}^{u}=\alpha_{a, 00,00}^{u} z_{a}+\alpha_{a, 00,10}^{u}$. The right hand side must be zero if the polarizability is symmetrical, but the left hand side is not found to be zero in practice: applying an electric field to a molecule causes the local charges to change. Substituting $l=0$ into equation (12) gives similar problems, and $l=1$ gives $\Pi_{a, 10,20}^{u}=\alpha_{a, 10,10}^{u}\left(2 z_{a}\right)+\alpha_{a, 10,20}^{u}$, where both polarizabilities on the right were obtained in the previous step, but in practice the calculated $\Pi_{a, 10,20}^{u}$ does not satisfy this equation. Finally, if the molecule has lower symmetry, applying potentials with the same $l$ but different $m$ can produce unsymmetrical polarizabilities $\alpha_{a, l m, l m^{\prime}}^{u} \neq \alpha_{a, l m^{\prime}, l m}^{u}$.

Our solution to this problem is a 'localisation' or 'symmetrisation' scheme which modifies the local multipole responses $\Pi_{a}^{u}$ to give new values $\Pi_{a}$. These are chosen such that the local polarizabilities $\alpha_{a}$, calculated from $\Pi_{a}$ using equation (9) (without the $u$ superscripts), are symmetrical and consistent. This is done by re-expressing part of the multipole response of each region $a$ as a series of multipole responses at neighbouring regions $b$, without changing the overall multipole response of the molecule. If a multipole response of $a$ is calculated to be $\Pi_{a, \lambda, \lambda^{\prime}}^{u}$, and a part $\Pi_{a \rightarrow b, \lambda, \lambda^{\prime}}$ of this is re-expanded about centre $b$, this changes the multipole responses $\Pi_{b, \lambda^{\prime \prime}, \lambda^{\prime}}^{u}$ of $b$, for all $l^{\prime \prime} \geq l$, by an amount $\Pi_{a \rightarrow b, \lambda, \lambda^{\prime}} T_{\lambda^{\prime \prime}, \lambda}\left(\mathbf{r}_{a}-\mathbf{r}_{b}\right)$. The modified multipole response of $a$, including such 'transfers' of response from $a$ to $b$ and also from $b$ to $a$, is therefore

$$
\Pi_{a, \lambda, \lambda^{\prime}}=\Pi_{a, \lambda, \lambda^{\prime}}^{u}+\sum_{b} \sum_{\lambda^{\prime \prime}} \Pi_{b \rightarrow a, \lambda^{\prime \prime}, \lambda^{\prime}} T_{\lambda, \lambda^{\prime \prime}}\left(\mathbf{r}_{b}-\mathbf{r}_{a}\right)-\sum_{b} \Pi_{a \rightarrow b, \lambda, \lambda^{\prime}}
$$

The sum over $\lambda^{\prime \prime}$ is restricted to $l^{\prime \prime} \leq l$.

The multipole shifts $\Pi_{a \rightarrow b, \lambda, \lambda^{\prime}}$ are calculated in increasing order of $l^{\prime}$ and $l$, in parallel with the calculation of the local polarizabilities. For each applied potential $\lambda^{\prime}$, starting from 
$l^{\prime}=1$, multipole shifts are obtained separately for each response $\lambda$, for $l \leq l^{\prime}$, starting from $l=0$. In the case of a linear molecule considered above, the procedure starts with $l^{\prime}=1$, $m^{\prime}=0, l=0, m=0$, and equation (13) becomes

$$
\Pi_{a, 00,10}=\Pi_{a, 00,10}^{u}+\sum_{b} \Pi_{b \rightarrow a, 00,10}-\sum_{b} \Pi_{a \rightarrow b, 00,10} .
$$

The left hand side of this equation must be zero, so the charge shifts $\Pi_{b \rightarrow a, 00,10}$ are chosen such that the right hand side is zero, that is, some 'unwanted' charge response of region $a$ to the applied field is 'moved' to other regions, and vice versa, such that the modified charge response of each region is zero. The choice of charge shifts which satisfy the constraint (14) for all regions $a$ is not unique, and the method for choosing the charge shifts and other multipole shifts is addressed later. The charge shifts $\prod_{b \rightarrow a, 00,10}$ then affect the response $\Pi_{a, \lambda, 10}$ of the dipoles and higher multipoles $\lambda$ of region $a$, via the middle term on the right hand side of equation (13). This occurs because 'moving' a charge produces higher multipoles at the new centre.

After obtaining the charge shifts, dipole shifts $\prod_{b \rightarrow a, 10, \lambda^{\prime}}$ are chosen in an analogous way. The local dipole response $\Pi_{a, 10, \lambda^{\prime}}^{u}$ is obtained from direct calculation. The 'corrected' dipole response $\Pi_{a, 10, \lambda^{\prime}}$ is known from equation (9) (without the $u$ superscripts) and from the requirement that the polarizabilities are symmetrical. The charge shifts $\Pi_{b \rightarrow a, 00, \lambda^{\prime}}$ were found in the previous step, so the dipole shifts are the only undetermined quantities in equation (13) when $l=1$.

The only case that is not covered by the above procedure is the non-symmetry of local polarizabilities $\alpha_{a, \lambda, \lambda^{\prime}}^{u}$ with $l=l^{\prime}$. In this case, the 'corrected' local polarizability is taken to be the mean value, $\alpha_{a, \lambda, \lambda^{\prime}}=\left(\alpha_{a, \lambda, \lambda^{\prime}}^{u}+\alpha_{a, \lambda^{\prime}, \lambda}^{u}\right) / 2$, and the local multipole responses are modified, using suitable multipole shifts, to produce this result.

The complete computational procedure thus proceeds as follows.

1. Apply $l=1$ potentials and calculate the local multipole responses.

2. Apply charge shifts to remove the local charge responses; this will also modify the local dipole and higher responses.

3. Apply dipole shifts to make the dipole-dipole polarizabilities symmetrical, as just discussed; this will also modify higher responses.

4. Calculate all the local dipole polarizabilities (dipole-dipole, dipole-quadrupole, and higher if required) using the modified local responses. 
5. Apply $l=2$ potentials and calculate a new set of local multipole responses.

6. Repeat step 2 to remove the charge responses.

7. Apply dipole shifts to produce the 'correct' dipole responses, which are calculated using the local dipole-dipole polarizabilities and dipole-quadrupole polarizabilities from step 4 .

8. Apply quadrupole shifts to make the quadrupole-quadrupole polarizabilities symmetrical.

9. Calculate all the local quadrupole polarizabilities (quadrupole-quadrupole, quadrupoleoctopole, and higher if required).

10. For higher values of $l$, repeat step 5 , step 6 for the charge responses, step 7 for the dipole, quadrupole and all other responses of lower rank than $l$, step 8 for the responses of rank $l$, and step 9 to obtain the polarizabilities.

We now consider the multipole shifts. Regardless of the multipole components $\lambda$ and $\lambda^{\prime}$, the shifts must be chosen to satisfy a general version of equation (14),

$$
\Pi_{a, \lambda, \lambda^{\prime}}-\Pi_{a, \lambda, \lambda^{\prime}}^{u}=\sum_{b}\left(\Pi_{b \rightarrow a, \lambda, \lambda^{\prime}}-\Pi_{a \rightarrow b, \lambda, \lambda^{\prime}}\right)
$$

or, more succinctly,

$$
\Pi_{a}^{r}=\sum_{b}\left(\Pi_{b \rightarrow a}-\Pi_{a \rightarrow b}\right)
$$

where $\Pi_{a}^{r}=\Pi_{a}-\Pi_{a}^{u}$ is the required change in multipole response of region $a$, which is known, and the shifts $\Pi_{a \rightarrow b}$ are to be determined. To preserve symmetry, it is essential that a multipole shift from $a$ to $b$ is accompanied by an equal and opposite shift from $b$ to $a$, that is, $\Pi_{b \rightarrow a}=-\prod_{a \rightarrow b}$.

In our previous work [16], it was assumed that $\Pi_{b \rightarrow a}=0$ unless a covalent bond exists between $a$ and $b$. This gives a unique set of multipole shifts for simply connected molecules, because for 'end' regions $a$ with only one bonded neighbour $b$, equation (16) and the antisymmetry of $\Pi_{b \rightarrow a}$ gives $\Pi_{b \rightarrow a}=\Pi_{a}^{r} / 2$ and all other $\Pi_{b^{\prime} \rightarrow a}=0$; all shifts for the 'end' regions are now known, and the procedure is repeated as necessary for all remaining regions having only one bonded neighbour with unknown shifts. However, if the molecule contains aromatic rings or other non-simply-connected features, the solution is no longer unique, and it is also scientifically undesirable, and often ambiguous, to introduce the subjective notion of a covalent bond into the procedure.

In this paper we therefore define the multipole shift between regions $a$ and $b$ by analogy with a flow of fluid between connected containers of initially different pressures. A positive 


$$
\Pi^{r}(t)=X e^{\Lambda t} X^{T} \Pi^{r}(0)
$$

where $\Pi^{r}(t)$ is a vector containing the $N$ values of $\Pi_{a}^{r}(t)$ for the $N$ regions of the molecule; $\Lambda$ is a diagonal matrix, which contains the eigenvalues of a matrix $k$ having off-diagonal elements $k_{a b}$ and diagonal elements $k_{a a}=-\sum_{b} k_{a b} ; X$ is the matrix of normalised eigenvectors of $k$. If the conditions above hold, the eigenvalues are all negative, apart from a single zero eigenvalue. The total 'flow', or multipole shift from $b$ to $a$ is given by

$$
\Pi_{b \rightarrow a}=-\int_{0}^{\infty} k_{a b}\left(\Pi_{b}^{r}(t)-\Pi_{a}^{r}(t)\right) d t
$$

The choice of 'rate constant' in our previous work [16] corresponds to $k_{a b}=0$ when $a$ and $b$ are not bonded, and $k_{a b} \neq 0$ when $a$ and $b$ are bonded; the results do not depend on any of the individual nonzero $k_{a b}$ values in this case. In order to retain the reasonable behaviour of this method for simply connected molecules, and to extend it to other molecules, we now propose, somewhat in the spirit of the original DMA method [2], that $k_{a b}$ be made equal to the charge density at the $a b$ midpoint, $\rho\left(\left(\mathbf{r}_{a}+\mathbf{r}_{b}\right) / 2\right)$, but that if this midpoint is closer to a third atom than it is to $a$ and $b$, we put $k_{a b}=0$. As a result, in most molecules at their equilibrium geometries, $k_{a b}=0$ for pairs of atoms that are not covalently bonded. The results of our previous work are unchanged, but the method can now be straightforwardly extended to other molecules which are not simply connected. The molecules and computational methodology that we have chosen for this study are presented in the next section, followed by the results and conclusions. 


\section{METHODS}

In order to test the methodology, coupled Hartree-Fock (CHF) theory is used for calculating static polarizabilities, and time-dependent coupled Hartree-Fock (TDCHF) theory is used for calculating dynamic polarizabilities, which are needed to obtain dispersion coefficients. The systems chosen for study are water, carbon dioxide, the ring molecules oxirane $\left(\mathrm{C}_{2} \mathrm{H}_{4} \mathrm{O}\right)$ and benzene $\left(\mathrm{C}_{6} \mathrm{H}_{6}\right)$, and the all-trans conformers of the alkane series, $\mathrm{C}_{n} \mathrm{H}_{2 n+2}$, $n=2-6$. Based on our previous study of static polarizabilities [16], united atoms are chosen for the calculations. Each $\mathrm{CH}_{n}$ group, $n=1-3$, is treated as a united atom centered on the carbon nucleus, and water is treated as a single entity. The molecular geometries are optimised using Molpro [17] at the SCF level using the aug-cc-pVDZ basis set [18] and the highest point-group symmetry appropriate for the molecule.

The multipoles for each (united) atom are calculated from Stone's modified DMA algorithm [6], which was developed to overcome the large basis set dependence of the original DMA algorithm. The modified DMA method involves using atomic grids, similar to those used in density functional theory (DFT) calculations, to evaluate the multipole integrals involving diffuse basis functions where the sum of the exponents is less than a given threshold value. The original DMA procedure is used for larger exponents. Numerical tests using Dunning's aug-cc-pVXZ basis sets [18] for $\mathrm{C}_{2} \mathrm{H}_{6}(\mathrm{X}=\mathrm{D}, \mathrm{T})$ and $\mathrm{C}_{3} \mathrm{H}_{8}(\mathrm{X}=\mathrm{D})$ show that a smaller grid than that recommended by Stone [6] may be used, without loss of accuracy, for multipoles up to and including $l=3$. Therefore, for molecules containing more than four carbon atoms, grid 5 given by Treutler and Ahlrichs [19] is used, with the Becke partitioning scheme recommended by Stone.

Local polarizabilities and dispersion energy coefficients are calculated using multipoles up to $l=3$ for each united atom. The isotropic $\left(\bar{\alpha}_{1}\right)$ and anisotropic $\left(\Delta \alpha_{1}\right)$ components of the dipole-dipole polarizabilities and the isotropic component of the quadrupole-quadrupole polarizability $\left(\bar{\alpha}_{2}\right)$ and of the octopole-octopole polarizability $\left(\bar{\alpha}_{3}\right)$ are defined in terms of the real and imaginary components $(l, \kappa)$ of the spherical harmonics $(l, m)[20]$ as

$$
\bar{\alpha}_{l}=\frac{1}{2 l+1} \sum_{\kappa} \alpha_{l \kappa, l \kappa}
$$


and

$\Delta \alpha_{1}=\left[\frac{1}{2}\left(\left(\alpha_{10,10}-\alpha_{11 c, 11 c}\right)^{2}+\left(\alpha_{10,10}-\alpha_{11 s, 11 s}\right)^{2}+\left(\alpha_{11 c, 11 c}-\alpha_{11 s, 11 s}\right)^{2}\right)+3\left(\alpha_{10,11 c}^{2}+\alpha_{10,11 s}^{2}+\alpha_{11 c, 11 s}^{2}\right)\right]^{1 / 2}$.

Results for the original and modified DMA schemes are shown in table I, using three different basis sets: Dunning's aug-cc-pVDZ and aug-cc-pVTZ basis sets and the Sadlej pVTZ basis set [18]. The dependence of the polarizabilities on basis set for the modified DMA method is small, whereas the diffuse functions in the Sadlej basis set cause the polarizabilities calculated by the original DMA method to differ completely from the other two basis sets. The modified DMA method is therefore used in the rest of this work, and, given the relatively small differences between the basis sets, the smallest basis set (aug-cc-pVDZ) is used for most calculations, although some results obtained with the aug-cc-pVTZ basis set are also shown.

Dispersion coefficients are obtained from the TDCHF dynamic polarizabilities $\alpha_{a}(i \omega)$ using the Casimir-Polder integral,

$$
C_{d i s p ; \lambda_{1}, \lambda_{2}, \lambda_{3}, \lambda_{4}}^{a b}=\frac{1}{2 \pi} \int_{0}^{\infty} \alpha_{a, \lambda_{1}, \lambda_{2}}(i \omega) \alpha_{b, \lambda_{3}, \lambda_{4}}(i \omega) d \omega
$$

where $a$ and $b$ are united atoms of molecules $A$ and $B$. The dynamic polarizabilities are localised in the same way as the static polarizabilities. This integral is evaluated using five-point numerical quadrature [21]. In order to reduce the amount of data presented, spherically averaged $C_{6}, C_{8}$, and $C_{10}$ dispersion coefficients $\left(C_{6}^{0}, C_{8}^{0}, C_{10}^{0}\right)$ are reported:

$$
C_{n}^{0}=\sum_{l}\left(\begin{array}{c}
2 l+2 l^{\prime} \\
2 l
\end{array}\right)\left\langle C_{d i s p ; \lambda, \lambda, \lambda^{\prime}, \lambda^{\prime}}\right\rangle
$$

where $2 l+2 l^{\prime}+2=n$, and the average is over all $\kappa$ and $\kappa^{\prime}[21]$.

\section{RESULTS AND CONCLUSIONS}

The local polarizabilities of the ring molecules oxirane and benzene are calculated at $\mathrm{CHF}$ /aug-cc-pVDZ level using the methodology introduced in section II. Oxirane is treated as three united atoms centred at the $\mathrm{C}$ and $\mathrm{O}$ nuclei. Since two different types of bond are present in the oxirane ring, the calculated local polarizabilities depend on the 'rate constants' $k_{a b}$ associated with each type of bond, through the ratio $k_{C O} / k_{C C}$. If this ratio is 
assumed to be equal to the ratio of the charge densities at the $\mathrm{C}-\mathrm{O}$ and $\mathrm{C}-\mathrm{C}$ bond centres, then its value calculated from SCF/aug-cc-pVDZ charge densities is 0.854 . Polarizabilities calculated using this value are compared with other possible $K=k_{C O} / k_{C C}$ ratios between 0.05 and 20 in figure 1.

The results show that the dependence of local polarizabilities on the ratio of $k$ values is fairly small over a wide range of $K$ ratios. The isotropic polarizabilities do not become unphysically negative, and the relative size of the $\mathrm{CH}_{2}$ and $\mathrm{O}$ polarizabilities remains qualitatively the same, for all the $K$ values considered. A dipole polarizability of about 10 atomic units is found for $\mathrm{CH}_{2}$. This is a little smaller than for $\mathrm{CH}_{2}$ groups in hydrocarbons, see for example table I. Its anisotropy is greater, which may be caused by the strained bond angles at the carbon atoms in oxirane.

To demonstrate the convergence of the local polarizabilities in practice, point-to-point induction energies are calculated for oxirane using randomly chosen pairs of points located at least 2 van der Waals radii from every atom, and no further than 4 van der Waals radii from at least one atom. The method is identical to that previously described $[14,16]$. The RMS differences between 250500 directly calculated point-to-point induction energies, and corresponding multipolar induction energies obtained from the local polarizability representation, are calculated and summarised in table II. When local polarizabilities up to $l=3$ are used, the RMS difference, expressed as a percentage of the complete range of calculated induction energies, is less than $0.1 \%$. The corresponding RMS difference using molecular polarizabilities up to $l=3$, expanded about the geometrical centre of the ring, is $0.2 \%$. These results are similar to results published previously for trans-alkanes [16], which shows that the method introduced in this work for dealing with the ring geometry does not noticeably affect the accuracy of the results.

Table III shows polarizabilities for the $\mathrm{CH}_{2}$ and $\mathrm{O}$ groups in oxirane, as previously described, and also for the $\mathrm{CH}$ groups in benzene, rotated into a coordinate system in which the $\mathrm{x}$-axis points from $\mathrm{C}$ towards $\mathrm{H}$ and the $\mathrm{z}$-axis is parallel to the 6 -fold molecular rotation axis. The same 'rate constant' $k$ is used for all the $\mathrm{C}-\mathrm{C}$ bonds in benzene, and the results are independent of this $k$ value. Our method preserves the symmetry of the polarizabilities, i.e., all the $\mathrm{CH}$ groups in benzene have identical polarizabilities when they are rotated into the same coordinate system. This follows from the linear nature of the transformation from the local multipole responses to the local polarizabilities. The average dipole polarizabilities 
of the $\mathrm{CH}$ groups in benzene must be one-sixth of the total molecular dipole polarizability, but other symmetry-allowed polarizability components are not constrained to a particular value in this way.

The table shows that using the modified DMA method gives local polarizabilities which are more physically reasonable (the average polarizabilities are positive) and which depend less on basis set. This supports the use of the modified DMA method. From the modified DMA results, it is pleasing to note that the local dipole, quadrupole and octopole polarizabilities increase less quickly with increasing multipole rank than the isotropic molecular polarizabilities (for benzene, the molecular polarizabilities calculated with the same basis set are $\bar{\alpha}_{1}=67, \bar{\alpha}_{2}=1396, \bar{\alpha}_{3}=43400$ ). The anisotropy of the $\mathrm{CH}$ polarizabilities in benzene is relatively large, and reflects the relative ease with which electrons can be induced to move parallel to the plane of the aromatic ring, rather than perpendicular to it. This anisotropy should be taken into account when modelling Van der Waals interactions of aromatic molecules.

As described in section III, local dispersion energy coefficients have been calculated in the TDCHF approximation from imaginary-frequency-dependent local polarizabilities. Table IV and $\mathrm{V}$ show dispersion energy coefficients for the interaction of carbon dioxide and water, respectively, with hydrocarbon molecules from ethane to hexane. The calculations use $\mathrm{CH}_{n}$ united atoms for the hydrocarbons, the water molecule is treated as a single united atom, and in carbon dioxide each atom is treated separately. As an indication of the quality of the TDCHF results, values of $C_{6}$ calculated using pseudo-dipole oscillator strength distributions (DOSDs)[22-24] are compared with the molecular values obtained in the current work in table VI. It can be seen that for the $\mathrm{CO}_{2}$-hydrocarbon interaction, the current results are from $13.4 \%$ to $11.7 \%$ lower than the the DOSD results, while for the $\mathrm{H}_{2} \mathrm{O}$-hydrocarbon interactions the current results are $15.3 \%$ to $13.2 \%$ lower than the DOSD results.

The local dispersion energy coefficients reflect the promising results already obtained [16] for the local polarizabilities. It seems that isotropic $C_{6}, C_{8}$ and even $C_{10}$ dispersion energy coefficients calculated using this method will be transferable between chemically similar functional groups. For example, the spherically averaged $C_{6}$ dispersion energy coefficients take values between 55 and 60 atomic units for $\mathrm{CH}_{3}$-water, between 31 and 34 atomic units for $\mathrm{CH}_{3}-\mathrm{C}\left(\mathrm{CO}_{2}\right)$, and between 37 and 40 atomic units for $\mathrm{CH}_{3}-\mathrm{O}\left(\mathrm{CO}_{2}\right)$. As expected, the coefficients are generally larger for $\mathrm{CH}_{3}$ groups than for $\mathrm{CH}_{2}$ groups, and there also seems 
to be a small but consistent difference between the two types of $\mathrm{CH}_{2}$ group defined in the tables. The convergence properties of the local $C_{n}$ coefficients with increasing $n$ are also good. In general, the local isotropic $C_{8}$ coefficients are less than 30 times larger than the $C_{6}$ coefficients in atomic units, and the $C_{10}$ coefficients are less than 30 times the $C_{8}$ coefficients, whereas for the molecular coefficients shown in table VI, these ratios are larger, and they vary much more with the size of the molecule. Some caution is necessary in interpreting these results quantitatively, because the higher $C_{n}$ coefficients are not expected to be close to the basis set limit.

In table VII, calculated non-expanded dispersion energies for water-hydrocarbon and $\mathrm{CO}_{2}$-hydrocarbon interactions are compared with the summation of the corresponding multipole series up to dipole, quadrupole and octopole polarizabilities, using the local dispersion energy coefficients calculated in this work (including anisotropic coefficients not shown explicitly above) as well as the molecular dispersion energy coefficients. The non-expanded calculations are performed at the same level of monomer TDCHF theory, with the same basis set. Intermolecular separations and orientations are chosen at random, but in order to avoid any significant charge overlap effects, all geometries where two heavy atoms are separated by less than twice the sum of their van der Waals radii are not used. Geometries where all heavy atoms are separated by more than four times the sum of their respective van der Waals radii are also rejected, to avoid a large number of small dispersion energies. A total of 5000 intermolecular geometries are used.

By comparing previous results for induction energies (table VIII of reference [16]) with the results for water-alkane interactions in table VII, it can be seen that the multipole series for the dispersion energy converges more slowly than it does for the induction energy. Further, the results obtained by considering only dipole polarizabilities $\left(\mathrm{C}_{6}\right.$ dispersion energy coefficients) are not negative enough. Figure 2 shows that this discrepancy is quite consistent when atomic dispersion energy coefficients are used; the spread of values calculated with $l=1$ is sufficiently small that the possibility of empirically scaling the multipolar values to fit the calculated values could be considered. This is not the case for the molecular $l=1$ results, for which the spread is much larger. When quadrupole and octopole polarizabilities are included in calculating the local dispersion energy coefficients, the series appears to converge towards the calculated values, and the RMS differences in table VII decrease. As might be expected, there is little or no evidence of convergence with increasing multipole rank 


\section{SUMMARY}

A method has been described for calculating the polarizabilities of atoms and united atoms within molecules. It uses the calculated response of local multipoles to an applied electrostatic potential, and adjusts these to give symmetrical, localised polarizabilities. This adjustment involves the transfer of multipoles between neighbouring atoms, which is done in a general way involving no fitting or subjective assumptions about bonding. The resulting polarizabilities appear to be transferable between functional groups in similar chemical environments.

The method should be suitable for use in more precise calculations of local polarizabilities, using larger basis sets and accounting for electron correlation. For Hartree-Fock and KohnSham calculations, a major contribution to the computer time is currently the calculation of the multipole integrals using the modified DMA method. It is likely that the computational effort could be reduced by treating all the basis set exponents using a DFT grid, rather than separating them into sums of exponents above and below a threshold value, and the effect of this simplification on the results should be investigated. The use of explicitly correlated methods to evaluate polarizabilities requires calculation of the response of the density matrix to each perturbation separately, which involves eight density calculations for dipole plus quadrupole perturbations, and a further seven for octopole perturbations. The total effort is therefore about an order of magnitude larger than a single-point energy calculation using the same theoretical method.

Calculating long-range energies from a multipole series requires truncation of the series at some multipole rank. From the results in this paper, it is suggested that including polarizabilities up to quadrupole gives the best compromise between accuracy and computational 
effort for both induction and dispersion energies. Local octopole polarizabilities do improve the results, and should probably be used for high-accuracy work on small molecules, but the improvement between $l=2$ and 3 is not so great as it is between $l=1$ and 2 , and the number and complexity of angular terms in the series increases significantly.

Local polarizabilities and dispersion energy coefficients calculated using this method should be suitable for applications such as fitting and modelling interactions between molecules, and molecular simulations of the gas, liquid and solid states. In condensed phases, non-additive interactions are important, and the calculated polarizabilities could also be used to evaluate local non-additive induction and dispersion energy coefficients.

\section{ACKNOWLEDGEMENTS}

The authors thank the Engineering and Physical Sciences Research Council for funding and the reviewer for making dispersion energy coefficients based on Refs [22-24] available. RJW thanks Prof. Anthony Stone and Dr. Alston Misquitta for valuable discussions. 
[1] A. J. Stone and A. J. Misquitta, Int. Rev. Phys. Chem. 26, 193 (2007).

[2] A. J. Stone and M. Alderton, Mol. Phys. 56, 1047 (1985).

[3] F. L. Hirschfeld, J. Mol. Struct. 130, 125 (1985).

[4] P. L. A. Popelier, Atoms in Molecules. An Introduction. (Prentice Hall, Harlow, 2000).

[5] J. F. Harrison, J. Chem. Phys. 119, 8763 (2003).

[6] A. J. Stone, J. Chem. Theory Comput. 1, 1128 (2005).

[7] A. J. Stone, Mol. Phys. 56, 1065 (1985).

[8] C. R. LeSueur and A. J. Stone, Mol. Phys. 78, 1267 (1993).

[9] C. R. LeSueur and A. J. Stone, Mol. Phys. 83, 293 (1994).

[10] J. Ángyán, G. Jansen, M. Loos, C. Hättig, and B. A. Heß, Chem. Phys. Letts. 219, 267 (1994).

[11] A. J. Stone, C. Hättig, G. Jansen, and J. G. Ángyán, Mol. Phys. 89, 595 (1996).

[12] M. in het Panhuis, P. L. A. Popelier, R. W. Munn, and J. G. Ángyán, J. Chem. Phys. 114, 7951 (2001).

[13] F. Dehez, C. Chipot, C. Millot, and J. Ángyán, Chem. Phys. Letts. 338, 180 (2001).

[14] G. J. Williams and A. J. Stone, Mol. Phys. 102, 985 (2004).

[15] A. J. Misquitta and A. J. Stone, J. Chem. Phys. 124, 024111 (2006).

[16] T. C. Lillestolen and R. J. Wheatley, J. Phys. Chem. A 111, 11141 (2007).

[17] H.-J. Werner, P. J. Knowles, R. Lindh, M. Schütz, et al., Molpro, version 2002.6, a package of ab initio programs (2003), see http://www.molpro.net.

[18] Basis sets were obtained from the Extensible Computational Chemistry Environment Basis Set Database, Version 02/02/06, as developed and distributed by the Molecular Science Computing Facility, Environmental and Molecular Sciences Laboratory which is part of the Pacific Northwest Laboratory, P.O. Box 999, Richland, Washington 99352, USA, and funded by the U.S. Department of Energy. The Pacific Northwest Laboratory is a multi-program laboratory operated by Battelle Memorial Institute for the U.S. Department of Energy under contract DE-AC06-76RLO 1830. Contact Karen Schuchardt for further information.

[19] O. Treutler and R. Ahlrichs, J. Chem. Phys. 102, 346 (1995).

[20] S. L. Price, A. J. Stone, and M. Alderton, Mol. Phys. 52, 987 (1984). 
[21] R. J. Wheatley, J. Comput. Chem. 29, 445 (2008).

[22] B. L. Jhanwar and W. J. Meath, Chem. Phys. 67, 185 (1982).

[23] B. L. Jhanwar and W. J. Meath, Mol. Phys. 41, 1061 (1980).

[24] D. J. Margoliash and W. J. Meath, J. Chem. Phys. 68, 1426 (1978). 
TABLE I: Local polarizabilities for the united atoms $\mathrm{CH}_{n}$ of the ethane and propane molecules, calculated using different local multipoles and different basis sets. The Dunning basis sets are abbreviated by aVDZ and aVTZ.

\begin{tabular}{|c|c|c|c|c|c|c|c|}
\hline $\bar{\alpha}_{1}$ & $\Delta \alpha_{1}$ & $\bar{\alpha}_{2}$ & $\bar{\alpha}_{3}$ & $\bar{\alpha}_{1}$ & $\Delta \alpha_{1}$ & $\bar{\alpha}_{2}$ & $\bar{\alpha}_{3}$ \\
\hline & \multicolumn{3}{|c|}{ Modified DMA } & \multicolumn{4}{|c|}{ Original DMA } \\
\hline \multicolumn{8}{|l|}{$\mathrm{C}_{2} \mathrm{H}_{6}$} \\
\hline $\mathrm{CH}_{3}$ aVDZ 13.67 & 1.65 & 98.39 & 953.5 & 13.67 & 1.65 & 97.16 & 972.7 \\
\hline $\mathrm{CH}_{3}$ Sadlej 13.65 & 1.65 & 99.74 & 1031.9 & 13.65 & 1.65 & 162.01 & 2763.6 \\
\hline $\mathrm{CH}_{3}$ aVTZ 13.70 & 1.67 & 101.01 & 980.2 & 13.70 & 1.67 & 95.80 & 979.8 \\
\hline \multicolumn{8}{|l|}{$\mathrm{C}_{3} \mathrm{H}_{8}$} \\
\hline $\mathrm{CH}_{3}$ aVDZ 13.56 & 2.85 & 100.24 & 1104.6 & 14.08 & 5.68 & 107.70 & 766.5 \\
\hline $\mathrm{CH}_{3}$ Sadlej 13.55 & 2.84 & 101.78 & 1207.9 & 9.26 & 26.59 & -197.72 & -2373.7 \\
\hline $\mathrm{CH}_{3}$ aVTZ 13.58 & 2.85 & 102.42 & 1239.8 & 13.79 & 2.52 & 103.25 & 1224.8 \\
\hline $\mathrm{CH}_{2}$ aVDZ 11.62 & 1.37 & 88.75 & 1027.3 & 10.58 & 6.59 & 63.83 & 1071.2 \\
\hline $\mathrm{CH}_{2}$ Sadlej 11.62 & 1.30 & 89.71 & 1086.9 & 20.20 & 48.07 & 986.72 & 16202.0 \\
\hline $\mathrm{CH}_{2}$ aVTZ 11.64 & 1.35 & 90.04 & 1128.9 & 11.21 & 0.93 & 62.65 & 652.0 \\
\hline
\end{tabular}


TABLE II: Induction energy results for oxirane. In the table, 'rms \%' refers to the root mean squared difference between calculated induction energies and the approximate values obtained by truncating the local or molecular multipole series at polarizabilities of rank $l$, expressed as a percentage of the complete range of calculated values. The 'max \%' value gives the largest such difference, and when it is negative, the multipole energy is below the calculated energy.

\begin{tabular}{|c|c|c|c|c|c|c|}
\hline \multicolumn{4}{|c|}{ Atomic } & \multicolumn{3}{|c|}{ Molecular } \\
\hline & $l=1$ & $l=2$ & $l=3$ & $l=1$ & $l=2$ & $l=3$ \\
\hline \multicolumn{7}{|c|}{ aug-cc-pVDZ } \\
\hline rms \% & 0.62 & 0.20 & 0.07 & 1.02 & 0.37 & 0.20 \\
\hline \multicolumn{2}{|c|}{$\max \%-22.19$} & -9.16 & -3.98 & -24.81 & -11.68 & 6.82 \\
\hline & & & & & & \\
\hline $\mathrm{rms} \%$ & 0.56 & 0.18 & 0.07 & 0.90 & 0.34 & 0.19 \\
\hline $\max \%$ & 1.17 & . & & רי 27 & 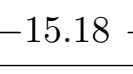 & \\
\hline
\end{tabular}

TABLE III: Isotropic and anisotropic local polarizabilities for united atoms of oxirane and benzene, calculated using different local multipoles and different basis sets.

\begin{tabular}{|c|c|c|c|c|c|c|c|c|}
\hline & \multicolumn{4}{|c|}{ Modified DMA } & \multicolumn{4}{|c|}{ Original DMA } \\
\hline & $\bar{\alpha}_{1}$ & $\Delta \alpha_{1}$ & $\bar{\alpha}_{2}$ & $\bar{\alpha}_{3}$ & $\bar{\alpha}_{1}$ & $\Delta \alpha_{1}$ & $\bar{\alpha}_{2}$ & $\bar{\alpha}_{3}$ \\
\hline \multicolumn{9}{|c|}{$\left(\mathrm{CH}_{2}\right)_{2} \mathrm{O}$} \\
\hline $\mathrm{CH}_{2}$ aVDZ & 10.47 & 6.26 & 67.86 & 584.24 & 9.91 & 6.80 & 82.25 & 496.72 \\
\hline $\mathrm{CH}_{2}$ aVTZ & 10.49 & 6.23 & 69.97 & 676.12 & 10.10 & 7.58 & 69.40 & 927.06 \\
\hline $\mathrm{O} \quad \mathrm{aVDZ}$ & 5.53 & 4.49 & 20.33 & 171.40 & 6.64 & 4.20 & 4.44 & -287.80 \\
\hline O aVTZ & 5.65 & 4.44 & 22.57 & 203.28 & 6.42 & 4.69 & 22.49 & -111.56 \\
\hline
\end{tabular}

CH aVDZ $11.235 .62 \quad 50.54818 .06 \quad 11.23 \quad 12.9166 .48 \quad 2124.42$ 
TABLE IV: Spherically averaged local dispersion energy coefficients for hydrocarbon- $\mathrm{CO}_{2}$ interactions. The notation $\mathrm{CH}_{2}(\mathrm{~A})$ denoted a functional group that is bonded to one or two methyl groups; $\mathrm{CH}_{2}(\mathrm{~B})$ is bonded to two other $\mathrm{CH}_{2}$ groups.

\begin{tabular}{rccccc}
\hline & $\mathrm{C}_{2} \mathrm{H}_{6}$ & $\mathrm{C}_{3} \mathrm{H}_{8}$ & $\mathrm{C}_{4} \mathrm{H}_{10}$ & $\mathrm{C}_{5} \mathrm{H}_{12}$ & $\mathrm{C}_{6} \mathrm{H}_{14}$ \\
\hline \hline $\mathrm{C}_{6} \mathrm{CH}_{3}-\mathrm{C}$ & 31.9 & 32.3 & 33.2 & 34.0 & 32.9 \\
$\mathrm{C}_{8} \mathrm{CH}_{3}-\mathrm{C}$ & 829 & 869 & 885 & 881 & 838 \\
$\mathrm{C}_{10} \mathrm{CH}_{3}-\mathrm{C}$ & 19700 & 17700 & 20700 & 15900 & 20700 \\
\hline $\mathrm{C}_{6} \mathrm{CH}_{2}(\mathrm{~A})-\mathrm{C}$ & - & 25.8 & 25.5 & 24.2 & 24.9 \\
$\mathrm{C}_{8} \mathrm{CH}_{2}(\mathrm{~A})-\mathrm{C}$ & - & 613 & 675 & 634 & 678 \\
$\mathrm{C}_{10} \mathrm{CH}_{2}(\mathrm{~A})-\mathrm{C}$ & - & 16700 & 17000 & 23600 & 19800 \\
\hline $\mathrm{C}_{6} \mathrm{CH}_{2}(\mathrm{~B})-\mathrm{C}$ & - & - & - & 28.0 & 28.1 \\
$\mathrm{C}_{8} \mathrm{CH}_{2}(\mathrm{~B})-\mathrm{C}$ & - & - & - & 816 & 741 \\
$\mathrm{C}_{10} \mathrm{CH}_{2}(\mathrm{~B})-\mathrm{C}$ & - & - & - & 20400 & 24000 \\
\hline $\mathrm{C}_{6} \mathrm{CH}_{3}-\mathrm{O}$ & 37.0 & 37.5 & 38.4 & 39.4 & 38.1 \\
$\mathrm{C}_{8} \mathrm{CH} \mathrm{CH}_{3}-\mathrm{O}$ & 928 & 973 & 989 & 984 & 936 \\
$\mathrm{C}_{10} \mathrm{CH}_{3}-\mathrm{O}$ & 20500 & 18100 & 21400 & 16000 & 21400 \\
\hline $\mathrm{C}_{6} \mathrm{CH}_{2}(\mathrm{~A})-\mathrm{O}$ & - & 30.0 & 29.6 & 28.2 & 28.9 \\
$\mathrm{C}_{8} \mathrm{CH}_{2}(\mathrm{~A})-\mathrm{O}$ & - & 684 & 753 & 707 & 756 \\
$\mathrm{C}_{10} \mathrm{CH}_{2}(\mathrm{~A})-\mathrm{O}$ & - & 17400 & 17400 & 24800 & 20400 \\
\hline $\mathrm{C}_{6} \mathrm{CH}_{2}(\mathrm{~B})-\mathrm{O}$ & - & - & - & 32.4 & 32.6 \\
$\mathrm{C}_{8} \mathrm{CH}_{2}(\mathrm{~B})-\mathrm{O}$ & - & - & - & 913 & 830 \\
$\mathrm{CH}_{2}(\mathrm{~B})-\mathrm{O}$ & - & - & - & 20900 & 24900 \\
\hline
\end{tabular}


TABLE V: Spherically averaged local dispersion energy coefficients for hydrocarbon- $\mathrm{H}_{2} \mathrm{O}$ interactions. The notation is the same as in table IV.

\begin{tabular}{rccccc}
\hline & $\mathrm{C}_{2} \mathrm{H}_{6}$ & $\mathrm{C}_{3} \mathrm{H}_{8}$ & $\mathrm{C}_{4} \mathrm{H}_{10}$ & $\mathrm{C}_{5} \mathrm{H}_{12}$ & $\mathrm{C}_{6} \mathrm{H}_{14}$ \\
\hline $\mathrm{C}_{6} \mathrm{CH}_{3}-\mathrm{H}_{2} \mathrm{O}$ & 55.5 & 56.3 & 57.7 & 59.1 & 57.1 \\
$\mathrm{C}_{8} \mathrm{CH}_{3}-\mathrm{H}_{2} \mathrm{O}$ & 1517 & 1587 & 1615 & 1612 & 1534 \\
$\mathrm{C}_{10} \mathrm{CH}_{3}-\mathrm{H}_{2} \mathrm{O}$ & 39200 & 36000 & 41200 & 33100 & 40800 \\
\hline $\mathrm{C}_{6} \mathrm{CH}_{2}(\mathrm{~A})-\mathrm{H}_{2} \mathrm{O}$ & - & 44.9 & 44.4 & 42.3 & 43.3 \\
$\mathrm{C}_{8} \mathrm{CH}_{2}(\mathrm{~A})-\mathrm{H}_{2} \mathrm{O}$ & - & 1127 & 1231 & 1154 & 1232 \\
$\mathrm{C}_{10} \mathrm{CH}_{2}(\mathrm{~A})-\mathrm{H}_{2} \mathrm{O}$ & - & 32500 & 33200 & 43900 & 37800 \\
\hline $\mathrm{C}_{6} \mathrm{CH}_{2}(\mathrm{~B})-\mathrm{H}_{2} \mathrm{O}$ & - & - & - & 48.6 & 48.9 \\
$\mathrm{C}_{8} \mathrm{CH}_{2}(\mathrm{~B})-\mathrm{H}_{2} \mathrm{O}$ & - & - & - & 1482 & 1355 \\
$\mathrm{C}_{10} \mathrm{CH}_{2}(\mathrm{~B})-\mathrm{H}_{2} \mathrm{O}$ & - & - & - & 39800 & 45300 \\
\hline
\end{tabular}

TABLE VI: Spherically averaged molecular dispersion energy coefficients for hydrocarbon- $\mathrm{CO}_{2}$ and hydrocarbon- $\mathrm{H}_{2} \mathrm{O}$ interactions from this work and DOSD values for $C_{6}[22-24]$.

\begin{tabular}{rrccccc}
\hline & & $\mathrm{C}_{2} \mathrm{H}_{6}$ & $\mathrm{C}_{3} \mathrm{H}_{8}$ & $\mathrm{C}_{4} \mathrm{H}_{10}$ & $\mathrm{C}_{5} \mathrm{H}_{12}$ & $\mathrm{C}_{6} \mathrm{H}_{14}$ \\
\hline $\mathrm{CO}_{2}$ & $\mathrm{C}_{6}$ & 212 & 300 & 390 & 480 & 570 \\
& $\mathrm{DOSD}$ & 245 & 347 & 446 & 547 & 645 \\
& $\mathrm{C}_{8}$ & 11500 & 20700 & 34600 & 54000 & 80600 \\
& $\mathrm{C}_{10}$ & 560000 & 1250000 & 2720000 & 5450000 & 10430000 \\
\hline $\mathrm{H}_{2} \mathrm{O}$ & $\mathrm{C}_{6}$ & 111 & 158 & 204 & 251 & 299 \\
& $\mathrm{DOSD}^{2}$ & 131 & 186 & 239 & 293 & 345 \\
& $\mathrm{C}_{8}$ & 4700 & 8900 & 15600 & 25200 & 38500 \\
& $\mathrm{C}_{10}$ & 170000 & 430000 & 1040000 & 2240000 & 4530000 \\
\hline
\end{tabular}


TABLE VII: Dispersion energy results for hydrocarbon- $\mathrm{CO}_{2}$ and hydrocarbon- $\mathrm{H}_{2} \mathrm{O}$ interactions.

Notation is the same as in table II.

\begin{tabular}{|c|c|c|c|c|c|c|c|}
\hline & \multicolumn{3}{|c|}{ Atomic } & \multicolumn{3}{|c|}{ Molecular } \\
\hline & & $l=1$ & $l=2$ & $l=3$ & $l=1$ & $l=2$ & $l=3$ \\
\hline \multirow[t]{2}{*}{$\mathrm{C}_{2} \mathrm{H}_{6}-\mathrm{CO}_{2}$} & $\operatorname{rms} \%$ & 2.47 & 0.43 & 0.22 & 4.19 & 1.88 & 0.34 \\
\hline & $\max \%$ & 16.79 & 6.70 & 2.55 & 30.64 & -20.69 & 4.16 \\
\hline \multirow{2}{*}{$\mathrm{C}_{3} \mathrm{H}_{8}-\mathrm{CO}_{2}$} & rms \% & 2.34 & 0.45 & 0.32 & 4.25 & 2.03 & 1.00 \\
\hline & $\max$ & 17.37 & 5.31 & 3.71 & 29.23 & -23.59 & -15 \\
\hline \multirow{2}{*}{$\mathrm{C}_{4} \mathrm{H}_{10}-\mathrm{CO}_{2}$} & $\mathrm{rms} \%$ & 2.25 & 0.47 & 0.35 & 4.47 & 3.12 & 1.71 \\
\hline & $\max \%$ & 14.93 & 5.01 & 3.41 & 30.54 & -35.27 & -19 . \\
\hline \multirow[t]{2}{*}{$\mathrm{C}_{5} \mathrm{H}_{12}-\mathrm{CO}_{2}$} & $\mathrm{rms} \%$ & 2.17 & 0.36 & 0.20 & 5.02 & 4.47 & 3.30 \\
\hline & $\max \%$ & 16.80 & 5.12 & 2.23 & 31.78 & -64.01 & -5 \\
\hline \multirow[t]{2}{*}{$\mathrm{C}_{6} \mathrm{H}_{14}-\mathrm{CO}_{2}$} & $\mathrm{rms} \%$ & 2.21 & 0.51 & 0.37 & 5.51 & 6.56 & 4.74 \\
\hline & $\max \%$ & 18.28 & 5.91 & 3.87 & 33.79 & -85.70 & -62.8 \\
\hline \multirow[t]{2}{*}{$\mathrm{C}_{2} \mathrm{H}_{6}-\mathrm{H}_{2} \mathrm{O}$} & rms $\%$ & 3.09 & 0.50 & 0.27 & 3.45 & 1.26 & 0.35 \\
\hline & $\max \%$ & 27.53 & 6.15 & 3.07 & 25.38 & -11.33 & -4.73 \\
\hline \multirow[t]{2}{*}{$\mathrm{C}_{3} \mathrm{H}_{8}-\mathrm{H}_{2} \mathrm{O}$} & $\mathrm{rms} \%$ & 3.22 & 0.57 & 0.36 & 4.13 & 1.90 & 1.29 \\
\hline & $\max$ & 27.88 & 7.73 & 5 & 30.81 & -21.00 & 18 \\
\hline \multirow[t]{2}{*}{$\mathrm{C}_{4} \mathrm{H}_{10}-\mathrm{H}_{2} \mathrm{O}$} & $\mathrm{rms} \%$ & 2.77 & 0.57 & 0.39 & 4.17 & 2.89 & 1.75 \\
\hline & $\max \%$ & 24.97 & 6.22 & 4.22 & 31.57 & -40.27 & -18.82 \\
\hline \multirow[t]{2}{*}{$\mathrm{C}_{5} \mathrm{H}_{12}-\mathrm{H}_{2} \mathrm{O}$} & $\mathrm{rms} \%$ & 2.92 & 0.47 & 0.24 & 4.84 & 4.47 & 3.66 \\
\hline & $\max \%$ & 28.65 & 6.00 & 2.85 & 36.45 & -51 & $-:$ \\
\hline \multirow[t]{2}{*}{$\mathrm{C}_{6} \mathrm{H}_{14}-\mathrm{H}_{2} \mathrm{O}$} & & 2.36 & 0.50 & 0.33 & 4.43 & 5.62 & 4. \\
\hline & ma & 26.38 & 5.59 & 3.6 & 1.54 & -98.00 & \\
\hline
\end{tabular}


FIG. 1: Local polarizabilities of the $\mathrm{CH}_{2}$ united atoms and $\mathrm{O}$ atom of oxirane as a function of the different treatment of $\mathrm{CO}$ and $\mathrm{CC}$ bonds in the localisation procedure. Isotropic dipole polarizabilities are shown in (a), the polarizability anisotropies in (b), and isotropic quadrupole polarizabilities in (c). Polarizabilities obtained using the aug-cc-pVDZ and aug-cc-pVTZ basis sets are shown by squares and triangles respectively. $K$ is the $k_{C O} / k_{C C}$ ratio defined in the text. The vertical line denotes the recommended value, $K=0.854$.

FIG. 2: Dispersion energies for butane- $\mathrm{CO}_{2}$. The molecular (a) and local (b) multipolar dispersion energies are plotted against the calculated induction energies. Red plus symbols denote multipolar energies calculated using polarizabilities up to $l=1$, blue crosses use polarizabilities up to $l=2$, and black stars use polarizabilities up to $l=3$. 


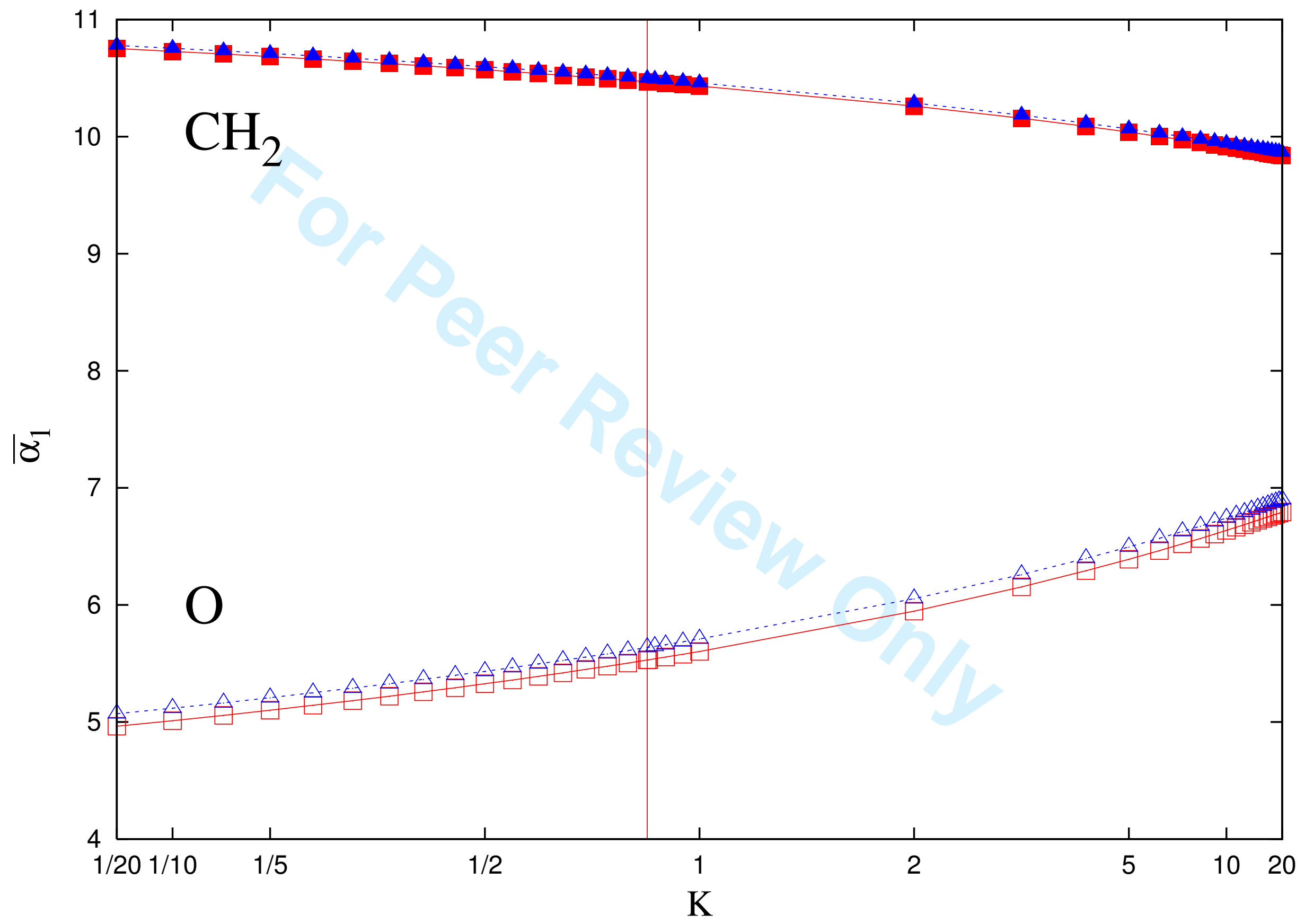

URL: http://mc.manuscriptcentral.com/tandf/tmph 


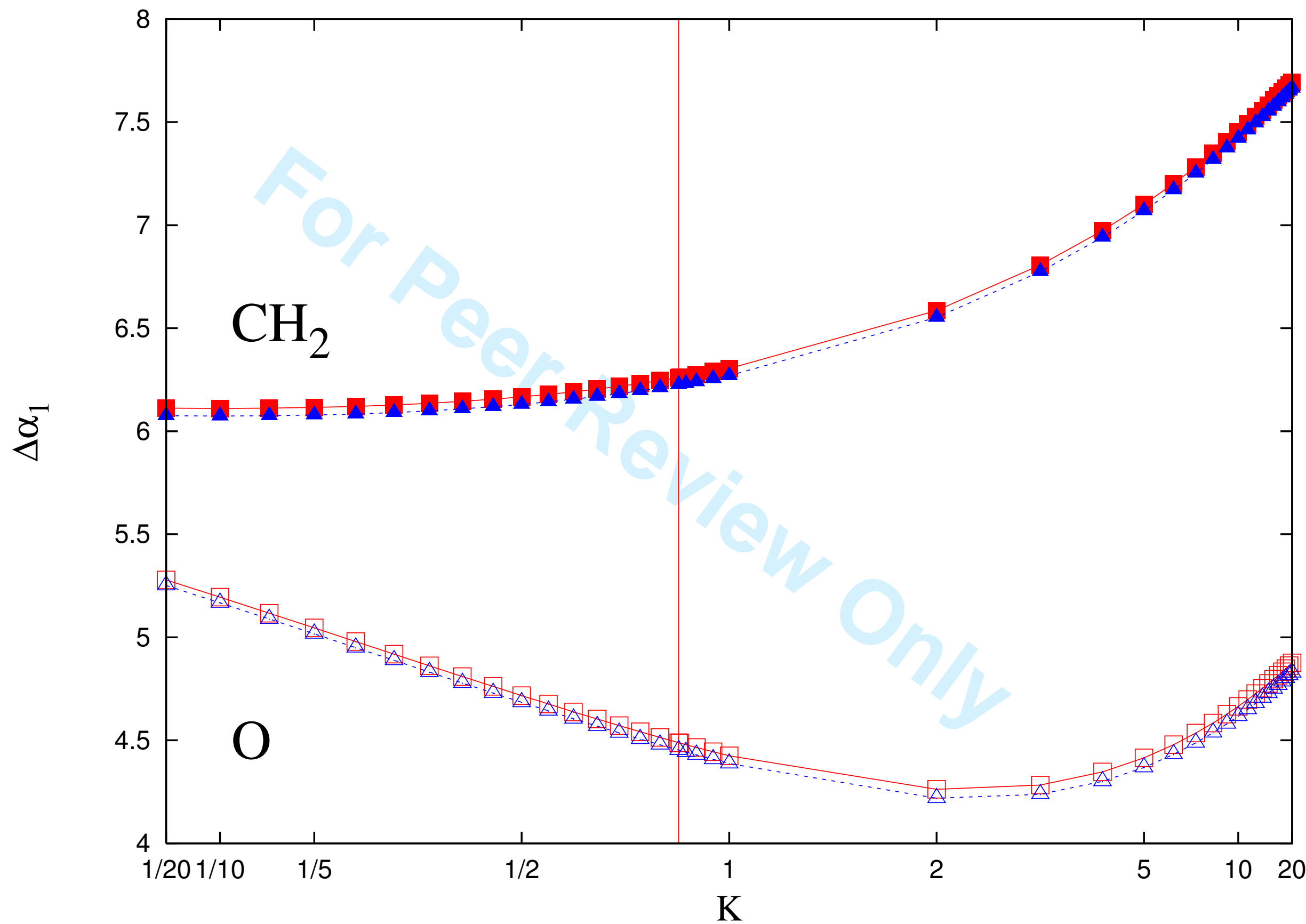

URL: http://mc.manuscriptcentral.com/tandf/tmph 


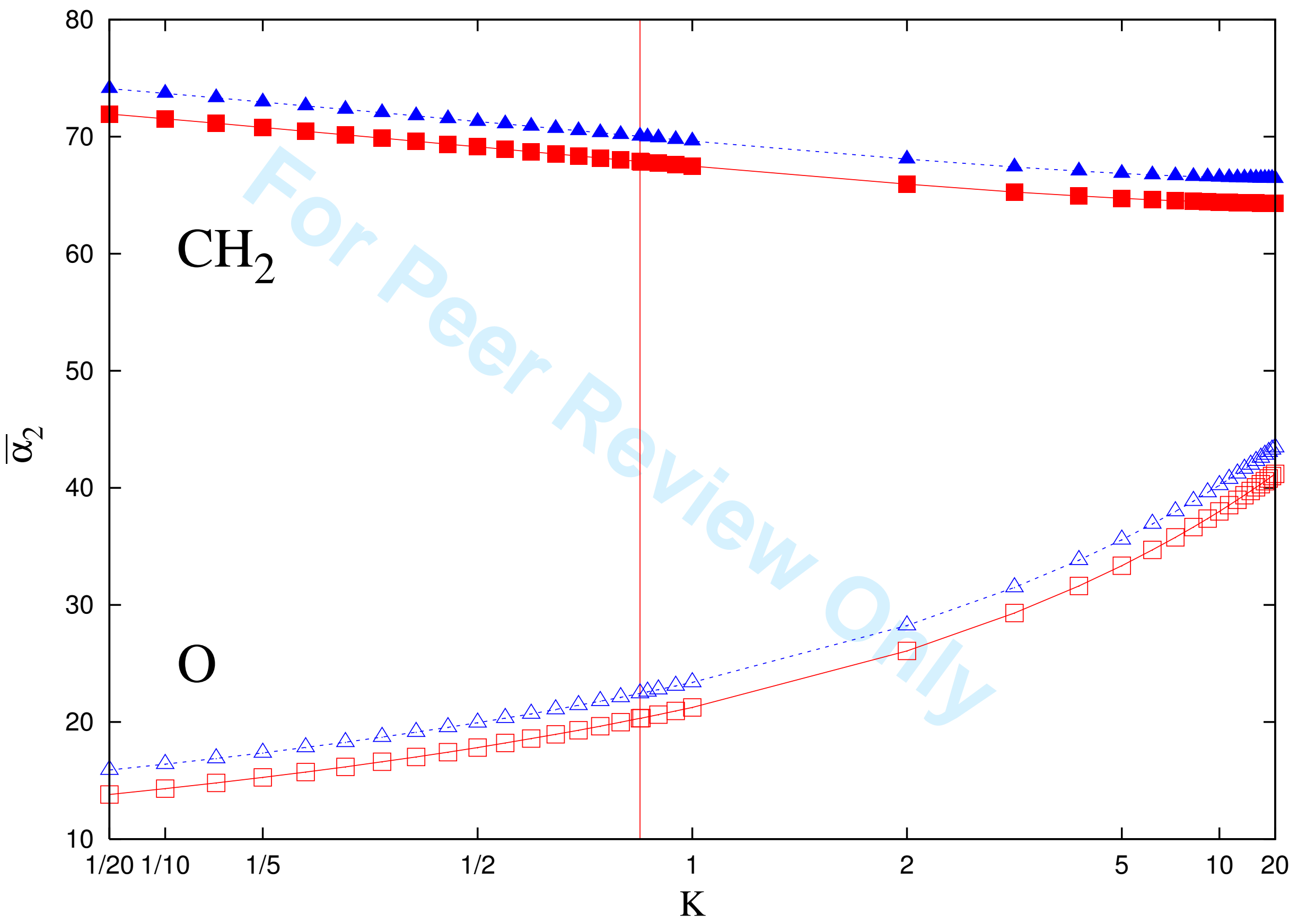

URL: http://mc.manuscriptcentral.com/tandf/tmph 


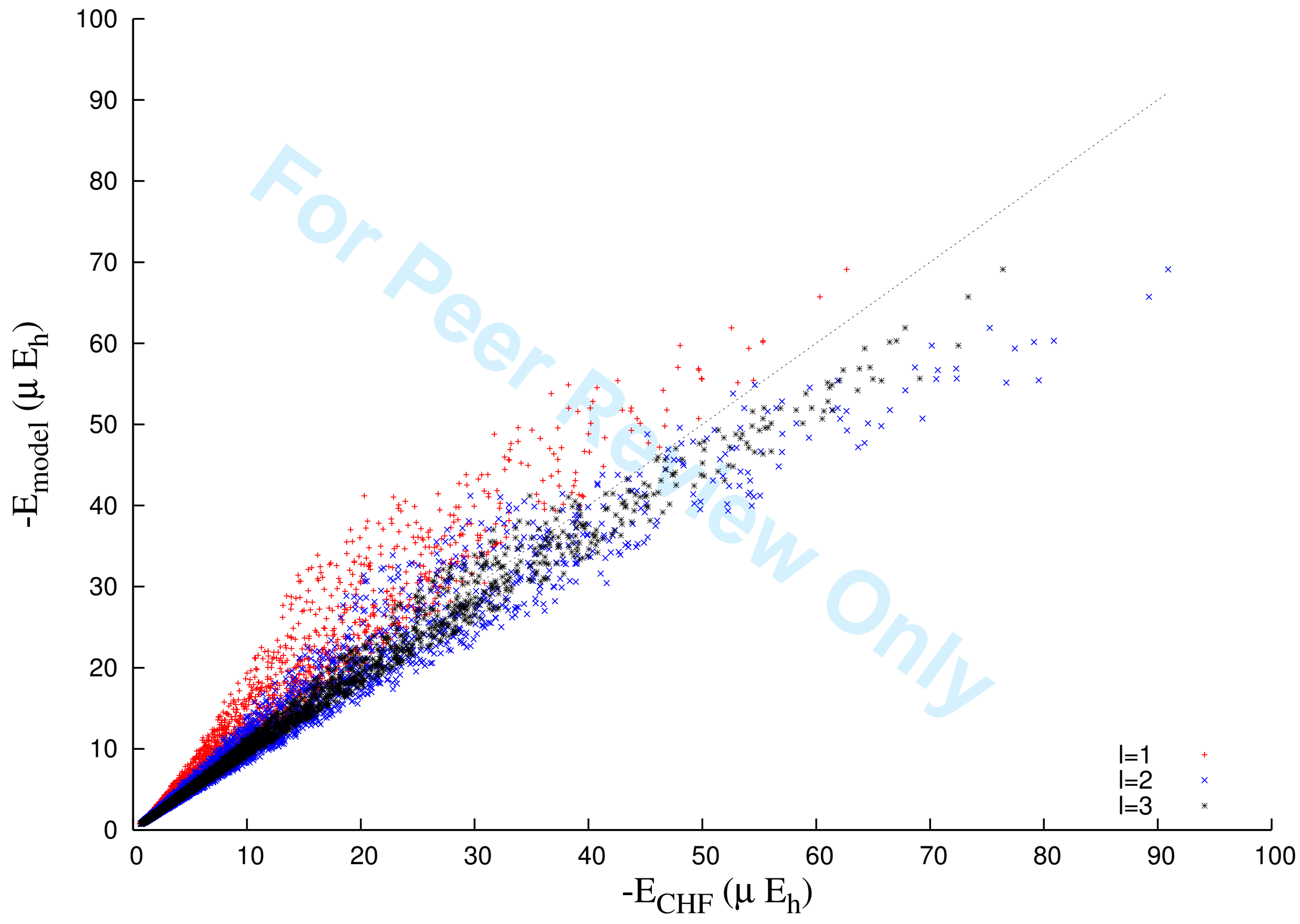

URL: http://mc.manuscriptcentral.com/tandf/tmph 


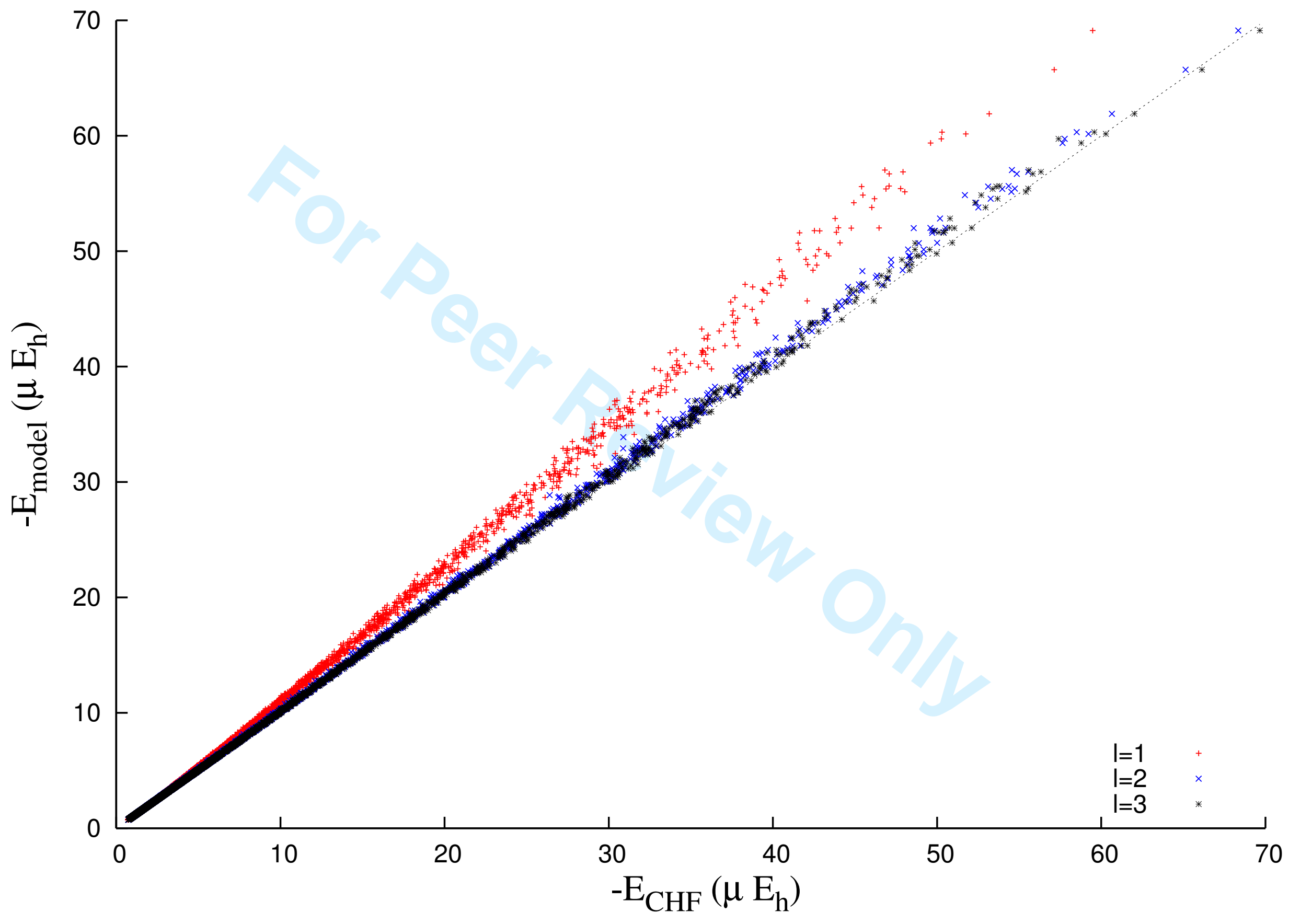

URL: http://mc.manuscriptcentral.com/tandf/tmph 